\title{
Les conditions d'émergence de communs porteurs de transformation sociale
}

Geneviève Fontaine, doctorante Université Paris Est Marne-la-Vallée, chargée de mission à l'Institut Godin - Amiens

Les crises environnementales, sociales, économiques et politiques actuelles peuvent constituer un contexte favorable à l'émergence d'innovations sociales à la fois réparatrices, permettant de rendre la transition moins douloureuse, mais aussi transformatives, s'inscrivant dans la mise en œuvre d'un autre modèle de développement (Levesque B., 2012). Dans le même temps, la pensée et le cadre d'analyse développés par Elinor Ostrom inspirent des initiatives et alimentent de très nombreux travaux, notamment en France, qui interrogent l'apport de cette approche des communs à l'analyse de la propriété (Orsi F., 2013), des institutions (Chanteau JP. et Labrousse A., 2013), du développement ou de l'Economie Sociale et Solidaire. Le pouvoir transformatif des communs en tant qu'actions collectives instituantes autour de ressources vécues comme vulnérables peut-il être questionné et renforcé par les analyses de l'innovation sociale?

En s'inscrivant dans une approche dite "forte " de l'innovation sociale comme vecteur de transformation sociale (Bouchard M., 2015), nous envisagerons dans un premier temps les apports croisés des approches institutionnalistes de l'innovation sociale et des communs afin de caractériser des communs orientés vers la transformation sociale.

Les conditions d'émergence de l'innovation sociale semblant influencer leur pouvoir transformatif (Richez-battesti, Besançon, 2015) et la question de l'émergence des communs étant présente dans les travaux le plus tardifs d'Ostrom, nous pourrons alors chercher à déduire de ce cadre d'analyse les conditions favorables à l'émergence de ces communs innovant socialement. Sans remettre en cause l'approche institutionnaliste des communs proposée par Ostrom et reprise par Coriat (2015), il s'agit, comme le suggère O. Weinstein (2015), de s'appuyer sur son analyse pour dégager les conditions favorables à l'émergence intentionnelle de communs potentiellement porteurs de transformation sociale.

\section{I - Approche institutionnaliste de l'innovation sociale et des communs : regards croisés}

L'analyse développée par E Ostrom sur les communs s'inscrit dans une réflexion plus large visant à comprendre les systèmes humains complexes. Ces travaux débouchent sur l'élaboration du cadre IAD (Institutionnal Analysis and Developpement) dont l'objectif est de définir un langage métathéorique, une " grammaire des institutions » permettant de croiser les données issues du terrain en provenance de champs disciplinaires variés afin d'analyser la diversité des situations d'interaction humaines. En référence explicite aux travaux d'Ostrom, nous proposons d'aborder les communs «comme des ensembles de ressources collectivement gouvernées, au moyen d'une structure de gouvernance assurant une distribution des droits entre les partenaires participant au commun (commoneurs) et visant à l'exploitation ordonnée de la ressource, permettant sa reproduction sur le long terme. » 
(Coriat, 2015, p. 38-39). En tant qu'action collective instituante, les communs se caractérisent donc par trois critères additionnels : 1) la production et la gestion par un groupe d'une ressource (considérée comme vulnérable) mise en commun ; 2) l'établissement, par un processus d'apprentissage collectif, de règles définissant des droits distribués (d'usage, d'utilisation..., etc.) qui régulent notamment l'accès à la ressource ; 3) enfin, la gouvernance collective de la ressource qui inclut la résolution des conflits.

Ostrom a ainsi défini un cadre d'analyse de la diversité des arrangements institutionnels permettant de caractériser des communs institués, d'étudier les conditions favorables à leur pérennité, à leur efficacité ou les processus d'évolution des règles les régissant - mais sans avoir exprimé clairement de visée normative et sans analyser leur potentiel transformatif. Or c'est précisément ce potentiel transformatif qui fonde l'approche institutionnaliste de l'innovation sociale quand elle est définie comme " une intervention initiée par des acteurs sociaux pour répondre à une aspiration, subvenir à un besoin, apporter une solution ou profiter d'une opportunité d'action afin de modifier des relations sociales, de transformer un cadre d'action ou de proposer de nouvelles orientations culturelles » (Bouchard M., 2015).

Quels sont les apports de l'approche institutionnaliste de l'innovation sociale à l'analyse des communs? A quelles conditions, le processus instituant du commun peut-il relever de l'innovation sociale?

Les travaux de I'Institut Godin (2013) ont permis de caractériser cette approche transformative de l'innovation sociale comme un processus socio-territorial :

L'innovation sociale est motivée par la volonté de répondre à un besoin social et/ou par une aspiration sociale (la visée d'un idéal social). Sa nouveauté réside dans la mise en œuvre de pratiques en rupture avec les pratiques habituelles dans un milieu donné. La dimension sociale prend forme dans un processus collectif marqué par des pratiques solidaires constitutives d'un ancrage territorial fort et de façon concomitante d'une gouvernance élargie et participative, se traduisant par un modèle économique pluriel. Ce processus aboutit à un résultat qui peut être une approche, un produit ou un service, se distinguant par son accessibilité et la logique de service qu'elle soustend. Processus et résultats sont porteurs d'impacts directs et de changements institutionnels multi-niveaux en ciblant les individus, les organisations, le territoire et les collectivités. Enfin, le déploiement de l'innovation sociale peut se faire de différentes manières : par essaimage, apprentissage et sélection.

Si l'on retrouve dans la construction d'un commun un processus collectif souvent territorialisé basé, selon Ostrom, sur des pratiques réciprocitaires s'ancrant dans différentes formes de proximité et engageant une gouvernance élargie pour déboucher sur une pratique en rupture avec les pratiques habituelles (le Marché ou l'Etat), le croisement avec l'approche socioterritoriale de l'innovation sociale nous amène à enrichir les critères d'analyse des communs sur plusieurs points pour en repérer le caractère transformatif. 
- au-delà du besoin social de protection d'une ressource vécue comme menacée ou vulnérable, la motivation de l'agir communs des commoneurs peut reposer sur une aspiration sociale aux changements institutionnels, à la transformation sociale. Cette aspiration sociale transformative constitutive de l'agir commun peut selon Manterola (2016) être le cadre cognitif du développement durable approché par les capabilités. En effet, l'innovation sociale dans son approche institutionnaliste et transformative peut être considérée comme facteur d'un développement local vu comme indissociable de l'accroissement général du bien-être et d'une promotion de l'idée de justice (Hillier et al., 2004). Or la recherche du bien-être et de la justice sociale de manière comparative, contextuellement située se retrouve dans l'approche par les capabilités du développement proposé par Sen : « Un développement durable se doit d'améliorer les capabilités de la génération actuelle sans compromettre le renforcement des capabilités des générations futures ». (Sen, 2000).

- la prise en compte de l'accessibilité dans le commun doit dépasser la recherche d'arrangements institutionnels permettant aux commoneurs de sécuriser leur accès à la ressource pour intégrer un objectif en termes d'empowerment (ou de capacitation) individuel et collectif.

- la réflexion sur les changements institutionnels recherchés et induits, aux niveaux territorial et organisationnel, amène à questionner la place et le rôle des pouvoirs publics locaux dans la construction des communs. Des communs porteurs de transformation sociale privilégieront une posture de co-construction avec des pouvoirs publics devenant commoneurs à part entière. D'une manière générale, le rôle de la diffusion dans l'analyse du pouvoir transformatif des innovations sociales enrichit la réflexion de certains auteurs pour qui développer les communs permettrait de diffuser les modes de pensée et de comportement de la coopération et ainsi « d'orienter la société vers une société des communs » (Defalvard ,2015). - la référence aux pratiques solidaires se traduisant notamment dans un modèle économique pluriel, amène à questionner le type de solidarité recherché et mis en œuvre au sein du commun pour privilégier une solidarité de type démocratique (Laville, 2014) et des formes d'échanges réciprocitaires ne renforçant pas la dissymétrie des positions sociales mais développant au contraire équitablement les capabilités (Manterola, 2016).

En tenant compte de ces remarques et apports, et en croisant les analyses d'Ostrom, de Sen et celles sur l'innovation sociale institutionnaliste, nous pouvons poser les éléments de caractérisation de communs de capabilité porteurs de transformation sociale :

\begin{tabular}{|c|c|c|}
\hline \multicolumn{3}{|c|}{ Critères additionnels de tous communs selon Ostrom } \\
\hline $\begin{array}{l}\text { 1) la production et la gestion en } \\
\text { commun d'une ressource } \\
\text { considérée comme vulnérable }\end{array}$ & $\begin{array}{l}\text { 2) l'établissement de droits } \\
\text { distribués de propriété qui } \\
\text { régulent l'accès à la ressource par } \\
\text { les commoneurs. }\end{array}$ & $\begin{array}{l}\text { 3) la gouvernance collective de la } \\
\text { ressource qui inclut la résolution } \\
\text { des conflits. }\end{array}$ \\
\hline \multicolumn{3}{|c|}{ Critères additionnels des communs de capabilité } \\
\hline \multicolumn{3}{|c|}{ 4) Une aspiration sociale au développement équitable des capabilités motive l'action collective } \\
\hline $\begin{array}{l}\text { 5) l'objectif est l'accessibilité réelle } \\
\text { à la ressource à partir d'une } \\
\text { finalité d'équité et une attention }\end{array}$ & $\begin{array}{l}\text { 6) un mode de gouvernance local } \\
\text { et délibératif associant les } \\
\text { personnes concernées (liberté }\end{array}$ & $\begin{array}{l}\text { 7) la ressource faisant l'objet d'un } \\
\text { commun de capabilité } \\
\text { (« ressource }\end{array}$ \\
\hline
\end{tabular}


portée aux plus démunis et procédurale) et qui repose sur une aux capabilités et fait l'objet d'un vulnérables. L'accessibilité se solidarité démocratique fondée jugement partagé d'utilité, de traduit notamment dans les droits sur la reconnaissance de l'autre désirabilité et d'un vœu de distribués qui régulent l'accès à la comme différent mais égal en dotation équitable construit par la ressource. dignité. délibération.

8) Des pouvoirs publics qui, en sus de la reconnaissance méta-institutionnelle des règles du communs (Ostrom, 1990), favorisent la liberté procédurale des acteurs et adoptent une posture de co-construction.

Nous définissons ainsi une version " forte " des communs comme vecteur de transformation sociale à l'image de la définition « forte » de l'innovation sociale portée par le CRISES.

Ainsi, lorsque face à la raréfaction de l'offre de service de santé (désertification médicale), les acteurs du médico-social du canton de Lorris (région Centre) - à savoir une association d'aide à domicile, un établissement pour personnes âgées dépendantes, une structure d'accueil et d'accompagnement de personnes en situation de handicap, les professionnels de santé, la pharmacie - se mobilisent avec les pouvoirs publics locaux pour préserver et gérer cette ressource dans une logique de défense du droit d'accès aux soins en définissant des arrangements institutionnels (un parcours sécurisé du médicament permettant de pallier au manque d'infirmier sur le territoire par exemple) négociés avec les instances de régulation, nous pouvons identifier une démarche de construction d'un commun de capabilité autour de la santé potentiellement porteur de transformation sociale. Les acteurs de Lorris se revendiquent à la fois des communs et des Pôles Territoriaux de Coopération économique et en ce sens, leur dynamique relève également d'un processus d'innovation sociale.

Si le processus instituant du commun peut relever de l'innovation sociale, on peut aussi appréhender l'innovation sociale comme un commun en construction (Emin, 2016) et l'analyse des communs de capabilité peut enrichir à son tour l'approche socio-territoriale de l'innovation sociale en termes de pouvoir transformatif.

Les communs de capabilité sont des communs ouverts au sens où de nouveaux commoneurs doivent pouvoir en permanence avoir accès à la ressource mais aussi au processus instituant (liberté procédurale de Sen). Cela invite à réfléchir l'accessibilité de manière transversale et permanente dans le processus d'innovation sociale: pas uniquement en termes de participation à la gouvernance et de résultats pour les bénéficiaires mais aussi en termes d'inclusion de nouvelles parties prenantes tout au long du processus d'élaboration et de diffusion.

Mais l'apport essentiel de l'analyse des communs se situe dans l'introduction (déjà présente chez J Commons) d'une réflexion sur la propriété en termes de faisceau de droits construits et distribués entre les commoneurs. Si le processus d'innovation social peut contribuer au développement local (Klein, 2014) c'est parce qu'il génère un nouvel actif territorial et collectif en révélant et combinant des ressources jusqu'ici non mobilisées, voire vécues comme des handicaps. La question de la construction, au cours du processus d'innovation sociale, d'un 
ensemble de règles définissant les droits (usage, utilisation, gestion, cession, aliénation) sur cet actif et de leur distribution entre les parties-prenantes actuelles et futures devient alors essentielle pour réfléchir la diffusion et le pouvoir transformatif de l'innovation sociale. Qui possède ce droit de diffusion ? Selon quelles règles ? Comment ont-elles été construites ? En quoi influencent-elles l'ampleur des impacts et des changements institutionnels induits ? Voir l'innovation sociale comme un commun en construction permet d'interroger les risques d'enclosures et d'appropriation de la ressource-actif générée en commun, ce qui rejoint les analyses sur le risque d'isomorphisme des dynamiques d'innovation sociale vis-à-vis des pratiques de marché (Emin, 2016).

Les apports croisés de l'analyse des communs, de l'approche institutionnaliste de l'innovation sociale et d'une conception du développement territorial qui dépasse la question des besoins pour appréhender celle des libertés (Sen) semblent donc féconds pour chercher à accroitre le pouvoir transformatif des actions collectives instituantes territorialisées. Or, il ressort des analyses institutionnalistes de l'innovation sociale que les conditions d'émergence de la dynamique collective conditionnent le processus d'innovation sociale dans son ensemble (Richez-Battesti, 2015) et en particulier celui de sa diffusion (Besançon, 2015). Nous devons donc chercher à dégager les conditions favorables à l'émergence intentionnelle de communs potentiellement porteurs de transformation sociale.

II-Questionner les conditions d'émergence des communs porteurs de transformation sociale.

L'analyse d'Ostrom ne se limite pas, comme on le dit souvent, à la recherche des conditions de réussite de l'action collective d'un groupe permettant d'assurer la production, l'usage, la préservation voire le développement de la ressource par l'élaboration d'arrangements.

Dès 1998, elle définit ainsi des variables clés qui expliquent le degré de capacité d'auto-organisation d'un collectif. En 2005, elle en propose la schématisation suivante :

Les " arènes d'action » sont vues comme l'unité de base de l'analyse institutionnelle au sens d'espace de production des règles (Chanteau \& Labrousse, 2013).

Dans sa description des attributs de la

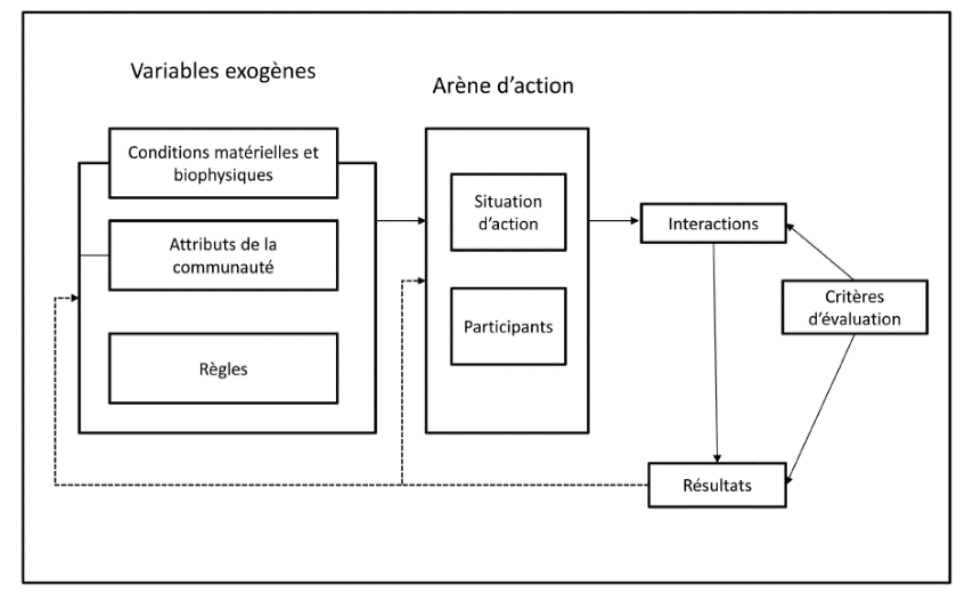

Cadre d'analyse IAD, adapté selon la schématisation d'Ostrom (2005, p.15) ressource (conditions matérielles et biophysiques), de la communauté et des participants, Ostrom présente des éléments qui relèvent de sa théorie des comportements et d'autres que l'on peut qualifier de structurels. S'y adjoignent des conditions favorables portant sur les 
règles exogènes qui nous donnent des indications sur la place et la posture des pouvoirs publics pour favoriser l'émergence de communs. Il est important de noter qu'Ostrom envisage ces variables comme étant interdépendantes et formant un système dynamique.

Nous avons regroupé ces conditions favorables à l'émergence de communs (Fontaine, 2016) issues directement ou indirectement du cadre d'analyse d'Ostrom dans la grille d'analyse suivante :

\begin{tabular}{|c|c|c|}
\hline \multicolumn{3}{|c|}{ Attributs structurels de la ressource } \\
\hline Vulnérabilité & \multicolumn{2}{|c|}{$\begin{array}{l}\text { L'idée de vulnérabilité renvoie à une dimension de protection du caractère durable de } \\
\text { la ressource en lien avec une solidarité intergénérationnelle mais aussi à la dimension } \\
\text { de sa non accessibilité effective et équitable. }\end{array}$} \\
\hline $\begin{array}{c}\text { Contexte } \\
\text { d'incertitude }\end{array}$ & \multicolumn{2}{|c|}{$\begin{array}{l}\text { Les contextes d'incertitude, les changements rapides dans les caractéristiques } \\
\text { physiques de la ressource, la non prévisibilité des bénéfices générés... incitent à la } \\
\text { coopération. }\end{array}$} \\
\hline $\begin{array}{l}\text { Accès à } \\
\text { l'information sur la } \\
\text { ressource }\end{array}$ & $\begin{array}{l}\text { La coopération se construira si } \\
\text { l'information sur les caractéristiques de la } \\
\text { ressource, sur son histoire et sur les } \\
\text { bénéfices locaux de son maintien ou } \\
\text { développement, est accessible et } \\
\text { partagée (Ostrom, 2011). }\end{array}$ & $\begin{array}{c}\text { => sera facilité par l'existence d'espaces } \\
\text { publics de proximité }\end{array}$ \\
\hline \multicolumn{3}{|c|}{$\begin{array}{l}\text { Attributs structurels des acteurs } \\
\end{array}$} \\
\hline Dépendance & \multicolumn{2}{|c|}{$\begin{array}{l}\text { La notion de dépendance n'est pas uniquement centrée sur la ressource faisant } \\
\text { l'objet du commun mais recouvre une triple interdépendance (Sabourin, 2003) des } \\
\text { acteurs vis-à-vis de la ressource, de la ressource par rapport à des ressources } \\
\text { complémentaires et des acteurs entre eux. }\end{array}$} \\
\hline $\begin{array}{l}\text { Forte préférence } \\
\text { pour le futur }\end{array}$ & \multicolumn{2}{|c|}{$\begin{array}{l}\text { C'est l'introduction du facteur temps qui permet la communication, le dialogue } \\
\text { constructif et l'apprentissage. La présence d'acteurs s'inscrivant dans une temporalité } \\
\text { longue est un facteur favorable à l'action collective. }\end{array}$} \\
\hline \multicolumn{3}{|c|}{ Attributs structurels du groupe } \\
\hline Proximité & \multicolumn{2}{|c|}{$\begin{array}{l}\text { Ostrom conserve l'idée de la taille réduite du groupe comme une prédisposition à } \\
\text { l'action collective mais face à la diversité des situations où paraissent pouvoir se } \\
\text { développer aujourd'hui des communs (Weinstein, 2015), c'est la notion de proximité } \\
\text { dans ses différentes acceptions (géographique, cognitive, de ressources) que nous } \\
\text { retenons (Bouba-Olga, 2008). }\end{array}$} \\
\hline \multirow[b]{2}{*}{ Homogénéité } & \multicolumn{2}{|c|}{$\begin{array}{l}\text { Renvoie davantage à l'idée de perception commune qu'à une caractéristique commune } \\
\text { intrinsèque des commoners. }\end{array}$} \\
\hline & $\begin{array}{l}\text { = perception commune de la ressource, } \\
\text { des autres usagers, des règles de } \\
\text { fonctionnement des institutions } \\
\text { extérieures. }\end{array}$ & $\begin{array}{c}=>\text { nécessite l'existence d'espaces } \\
\text { publics de proximité }\end{array}$ \\
\hline \multicolumn{3}{|c|}{ Variables issues de l'analyse comportementale } \\
\hline $\begin{array}{l}\text { Prise de conscience } \\
\text { des dépendances }\end{array}$ & $\begin{array}{c}=>\text { nécessite l'accès à l'information sur la } \\
\text { ressources, les autres, l'histoire ... }\end{array}$ & $\begin{array}{l}=>\text { nécessite l'existence d'espaces } \\
\text { publics de proximité }\end{array}$ \\
\hline $\begin{array}{l}\text { Capacité à fixer de } \\
\text { nouvelles règles }\end{array}$ & \multicolumn{2}{|c|}{$\begin{array}{l}\text { Le terme de " self governed " employé par Ostrom reflète, selon nous, un sentiment } \\
\text { de légitimité des acteurs à définir des règles qui rendent possible le développement du } \\
\text { commun. => dépend des règles externes et normes en vigueur }\end{array}$} \\
\hline
\end{tabular}




\begin{tabular}{|c|c|c|}
\hline \multirow[t]{2}{*}{ Expérience } & $\begin{array}{l}=\text { attitude positive vis-à-vis des } \\
\text { apprentissages }\end{array}$ & $\begin{array}{l}=\text { pratiques coutumières de coopération } \\
\text { ou d'entraide }\end{array}$ \\
\hline & \multicolumn{2}{|c|}{ => existence préalable de pratiques réciprocitaires } \\
\hline Confiance & \multicolumn{2}{|c|}{$\begin{array}{l}\text { La confiance est la condition de la décision initiale de coopérer. Elle repose pour Ostrom } \\
\text { sur la réputation des participants, sur l'existence d'un système de sanctions, au moins } \\
\text { informel, positives et négatives qui rejailliront sur la réputation et enfin sur une } \\
\text { anticipation positive de réciprocité néanmoins porteuse d'un risque de non-réciprocité. }\end{array}$} \\
\hline $\begin{array}{l}\text { Leader } \\
\text { charismatique }\end{array}$ & \multicolumn{2}{|c|}{$\begin{array}{l}\text { Ostrom souligne que les actions collectives façonnant les communs sont facilitées par } \\
\text { des leaders charismatiques ou par un noyau actif à l'interface entre le groupe et son } \\
\text { environnement. }\end{array}$} \\
\hline \multicolumn{3}{|c|}{ Règles et normes externes en vigueur } \\
\hline \multicolumn{3}{|c|}{$\begin{array}{l}\text { Influençant la vulnérabilité de la ressource en permettant ou même en organisant sa surexploitation } \\
\text { (Fattori, 2015) et son accessibilité. }\end{array}$} \\
\hline \multicolumn{3}{|c|}{ Permettant l'existence d'espaces publics de proximité afin de développer des schémas de pensée partagés. } \\
\hline \multicolumn{3}{|c|}{ Favorisant les prédispositions individuelles à la coopération } \\
\hline \multicolumn{3}{|c|}{ Favorisant la responsabilisation et l'autonomie } \\
\hline
\end{tabular}

On retrouve ici des conditions identifiées également comme favorables à l'innovation sociale et à sa diffusion : l'importance de la confiance, des intentions réciprocitaires, de l'attitude face aux apprentissages, de l'autonomie des acteurs et de la présence de leader mais peuton identifier, à partir des analyses institutionnalistes de l'innovation sociale et de l'approche par les capabilités du développement local, des conditions favorables spécifiques à l'émergence de communs dans leur version « forte " ?

\begin{tabular}{|c|c|}
\hline \multicolumn{2}{|r|}{ Attributs structurels de la ressource } \\
\hline \multirow[t]{2}{*}{$\begin{array}{c}\text { Une ressource } \\
\text { " sociale " } \\
\text { définie et } \\
\text { délimitée par } \\
\text { un processus } \\
\text { délibératif local }\end{array}$} & $\begin{array}{l}\text { La ressource mise en commun est sociale au sens où elle fait l'objet d'un jugement } \\
\text { partagé d'utilité, de désirabilité et d'un vœu de dotation équitable construit par la } \\
\text { délibération locale. L'accès effectif à cette ressource renforce les capabilités individuelles } \\
\text { et concoure au développement territorial durable dans une perspective de justice sociale } \\
\text { (Klein, 2014) }\end{array}$ \\
\hline & $\begin{array}{l}\text { => renforce la nécessité de l'existence préalable d'espaces publics de proximité ou d'un } \\
\text { rôle d'intermédiation entre les acteurs (Klein, 2014) pour renforcer en la proximité } \\
\text { spatiale et relationnelle avec une approche territorialisée mais aussi intersectorielle } \\
\text { (Levesque, 2016). }\end{array}$ \\
\hline \multicolumn{2}{|r|}{ Attributs structurels des acteurs } \\
\hline $\begin{array}{c}\text { Aspiration } \\
\text { sociale } \\
\text { transformative }\end{array}$ & $\begin{array}{l}\text { Les acteurs sont mus par une finalité qui dépasse la recherche d'un intérêt mutuel pour } \\
\text { chercher à agir sur les enjeux de société comme les inégalités ou le développement durable } \\
\text { (Bouchard, 2015). Cette aspiration peut être le développement équitable d'égales } \\
\text { capabilités pour chacun. Cette aspiration renforce la préférence forte pour le futur des } \\
\text { acteurs. }\end{array}$ \\
\hline $\begin{array}{c}\text { Responsabilité } \\
\text { ex-ante }\end{array}$ & $\begin{array}{l}\text { Les acteurs sont des "personnes capables " au sens de Ricoeur (2004) c'est-à-dire des } \\
\text { agents capables de ressentir et d'exercer une responsabilité ex-ante à la fois locale en tant } \\
\text { que citoyen d'un territoire et universelle vis-à-vis d'autrui et des générations futures. Cette } \\
\text { responsabilité ex-ante fonde un engagement des acteurs qui conditionne les processus } \\
\text { d'apprentissages collectifs et la diffusion de l'innovation sociale (Richez-Battesti, 2015) }\end{array}$ \\
\hline \multicolumn{2}{|r|}{ Attributs structurels du groupe } \\
\hline
\end{tabular}




\begin{tabular}{|c|c|}
\hline $\begin{array}{l}\text { Une proximité } \\
\text { de projet forte }\end{array}$ & $\begin{array}{l}\text { Le groupe se constitue autour de l'objectif d'agir sur les facteurs de conversion (Sen, 2010) } \\
\text { individuels, sociaux et environnementaux pour permettre aux individus un accès réel à la } \\
\text { ressource sociale. Cet objectif partagé dessine une proximité de projet forte qui permet } \\
\text { d'envisager une organisation polycentrique des communs de capabilité et leur imbrication } \\
\text { sur plusieurs niveaux. Elle peut participer au passage d'une identité territoriale partagée à } \\
\text { une conscience territoriale constitutive pour Klein (2014) de la construction sociale d'un } \\
\text { milieu dynamique apte à l'innovation sociale et au changement. }\end{array}$ \\
\hline \multicolumn{2}{|r|}{ Variables issues de l'analyse comportementale } \\
\hline $\begin{array}{c}\text { Solidarité } \\
\text { démocratique }\end{array}$ & $\begin{array}{l}\text { Les acteurs basent leurs relations réciprocitaires sur l'expression d'une solidarité fondée } \\
\text { sur la reconnaissance de l'autre comme différent mais égal en dignité. Recherche d'une } \\
\text { réciprocité émancipatrice par des actions collectives multi-partenariales entre acteurs } \\
\text { hétérogènes. }\end{array}$ \\
\hline \multicolumn{2}{|r|}{ Règles et normes externes en vigueur } \\
\hline \multicolumn{2}{|c|}{$\begin{array}{l}\text { Des pouvoirs publics qui soutiennent mais laisse aux acteurs la possibilité d'être créatifs (Klein, 2014) voir } \\
\text { qui développent leur capacité créatrice en adoptant une posture de partenaires, de co-production } \\
\text { renforçant ainsi le pouvoir transformatif du commun par la création de liens systémique avec l'innovation } \\
\text { publique (Bouchard, 2015). }\end{array}$} \\
\hline
\end{tabular}

\section{Eléments conclusifs et mise en perspective}

Les apports croisés de l'analyse des communs, de l'approche institutionnaliste de l'innovation sociale et d'une conception du développement territorial fondée sur les capabilités apparaissent comme une piste de recherche à la fois pour alimenter la réflexion sur le processus d'innovation sociale comme facteur de développement local et pour chercher à accroitre le pouvoir transformatif des dynamiques collectives territoriales et en particulier, en France, celle des Pôles Territoriaux de Coopération Economique (PTCE).

En les abordant comme des processus en cours de construction, la plupart des PTCE approchent plus ou moins des conditions qui les définissent comme des communs au sens d'Ostrom. Par contre, rares sont ceux qui réunissent les critères plus restrictifs des communs de capabilité qui supposent la mise en place de mécanismes rendant l'accès aux ressources équitables, notamment pour les personnes les plus vulnérables, et satisfaisant à une gouvernance délibérative entre les commoneurs (Defalvard et Fontaine, 2017). Lorsque c'est le cas, les PTCE sont structurés autour de l'accès à des biens fondamentaux tels l'emploi (Idesol), la santé (Lorris) ou directement autour d'une finalité de développement local durable (TETRIS). Cette grille d'analyse des communs de capabilité et des conditions favorables à leur émergence peut être utilisée pour guider la construction de PTCE afin d'en accroitre le potentiel transformatif en tant qu'écosystèmes territoriaux d'innovation sociale (Levesque, 2016). Par le biais de la conjugaison d'activités de recherche et de transfert (Chochoy,2015), c'est l'expérimentation en cours au sein du PTCE TETRIS (Transition Ecologique Territoriale par la Recherche et l'Innovation Sociale) sur le pays de Grasse et du PTCE de Lorris ; mais cela nous renvoie à la question récurrente du rôle et de la posture des chercheurs dans, avec ou pour ces dynamiques? 


\section{Bibliographie}

BESANCON E., CHOCHOY N. et GUYON T., 2013, L'innovation sociale. Du concept aux pratiques, Paris, Harmattan

BESANCON E. (2015), "La diffusion de l'innovation sociale, un processus de changement multiforme. Une illustration à partir d'une recyclerie", Sociologies pratiques 2015/2 (n³1), p. 31-40.

Entretien avec M.J BOUCHARD réalisé par FRAISSE L. (2015), "Concevoir l'innovation sociale dans une perspective de transformation", Sociologies pratiques 2015/2 ( $\left.N^{\circ} 31\right)$, p 9-14.

BOUBA-OLGA O. et GROSSETTI M. (2008), "Socio-économie de proximité", Revue d'Economie Régionale \& Urbaines, 2008/3 (octobre), p.311-328.

CHANTEAU JP, LABROUSSE A. (2013), "L'institutionnalisme méthodologique d'Elinor Ostrom : quelques enjeux et controverses ", ", Revue de la régulation, $n^{\circ} 14$, dossier : Autour d'Ostrom : communs, droits de propriété et institutionnalisme méthodologique

CHOCHOY N. (2015), "Médiation entre acteurs de la recherche et acteurs économiques : originalité des pratiques de recherche et de transfert de l'Institut Godin", Sociologies pratiques 2015/2 (N³1), p. 75- 83.

CORIAT B. (dir.) (2015), " Communs fonciers et communs intellectuels. Comment définir un commun ?» in Le retour des communs - La crise de l'idéologie propriétaire, Les liens qui libèrent, p23-50.

DEFALVARD H. (2015), La révolution de l'économie [en 10 leçons], Les éditions de l'Atelier, 190 p.

DEFALVARD H. et FONTAINE G. (2017), « La place des pouvoirs publics dans les PTCE en tant que communs sociaux », communication au $27^{\text {ième }}$ colloque de l'ADDES, 26 janvier2017, Paris.

EMIN S. (2016), "Innovation sociale, un détour par les communs", Communication lors du XVle colloque du RIUESS, Montpellier, mai 2016.

HILLIER J et al. (2004), "Trois essais sur le rôle de l'innovation sociale dans le développement territorial", Géographie, économie, société 2004/2 (Vol. 6), p. 129-152.

KLEIN J.L. (2014), "Innovation sociale et développement territorial", in Juan-Luis Klein et al., L'innovation sociale, ERES "Sociologie économique", 2014, p.115-142

LAVILLE J-L (2014), « Innovation sociale, économie sociale et solidaire, entrepreneuriat social. Une mise en perspective historique ", in L'innovation sociale, J-L Klein, J-L Laville et F. Moulaert (dir.), p45-80, Erès.

LEVESQUE B. (2012), "Crises et innovation sociale", http://www.eurozine.com/articles/2012-06-05-levesque$\underline{\text { fr.html }}$

LEVESQUE B. (2016), "Economie sociale et solidaire et entrepreneuriat social: vers quels nouveaux écosystèmes?", Revue Interventions Economiques [en ligne], 54/2016, mis en ligne le 01 mars 2016, consulté le 13 juillet 2016.

MANTEROLA J.J. (2016), "Vers un système territorial d'innovation sociale", Working Papers N¹8, Cahiers du Centre Emile Durkheim, Sciences Po Bordeaux, juin 2016.

ORSI F. (2013), "Elinor Ostrom et les faisceaux de droits : I'ouverture d'un nouvel espace pour penser la propriété commune", Revue de la régulation, $\mathrm{n}^{\circ} 14$, dossier : Autour d’Ostrom : communs, droits de propriété et institutionnalisme méthodologique. 
OSTROM E. (2011), « Plaidoyer pour la complexité », Ecologie \& politique 2011/1, n41, p 111-121.

RICHEZ-BATTESTI N. (2015), "Les processus de diffusion de l'innovation sociale : des arrangements institutionnels diversifiés ?", Sociologies pratiques 2015/2 (n³1), p. 21-30.

SEN A (2000), Un nouveau modèle économique. Développement, justice, liberté, Editions Odile Jacob, Paris, 2000.

WEINSTEIN O (2015), "Comment se construisent les communs : question à partir d'Otsrom », in Le retour des communs- La crise de l'idéologie propriétaire, CORIAT B. (dir.), Les liens qui libèrent, p 69-86. 